\section{$\underset{\substack{\text { hommes } \\ \text { \& migrations }}}{ }$}

\section{Hommes \& migrations}

Revue française de référence sur les dynamiques

migratoires

1308 | 2014

Les Paris des migrants

\title{
Le gwoka, patrimoine culturel immatériel
}

\section{François Bensignor}

\section{(2) OpenEdition \\ Journals}

\section{Édition électronique}

URL : http://journals.openedition.org/hommesmigrations/3022

DOI : 10.4000/hommesmigrations.3022

ISSN : 2262-3353

\section{Éditeur}

Musée national de l'histoire de l'immigration

\section{Édition imprimée}

Date de publication : 1 octobre 2014

Pagination : 184-189

ISBN : 978-2-919040-29-2

ISSN : $1142-852 X$

\section{Référence électronique}

François Bensignor, «Le gwoka, patrimoine culturel immatériel », Hommes \& migrations [En ligne], 1308 | 2014, mis en ligne le 26 juin 2015, consulté le 24 septembre 2020. URL : http:// journals.openedition.org/hommesmigrations/3022; DOI : https://doi.org/10.4000/ hommesmigrations.3022 


\title{
LE GWOKA, PATRIMOINE CULTUREL IMMATÉRIEL
}

\author{
FRANÇOIS BENSIGNOR
}

A ujourd'hui le gwoka fait la fierté des Guadeloupéens. Ses jeux de tambour, paroles chantées, pratiques de la danse font société au point que l'identité culturelle de la Guadeloupe ne saurait s'en passer. Pourtant, cette pratique artistique et sociale aurait pu disparaitre au milieu du XXe siècle, victime d'une certaine vindicte populaire. La "bonne" société créole n'avait alors que mépris pour cette mizik a vié nèg (musique de sauvages). Or le chemin parcouru en faveur de sa réhabilitation aboutit à l'inscription du gwoka sur la liste représentative du patrimoine culturel immatériel de l'humanité de l'Unesco (PCI). L'annonce en a été faite par le Comité du PCI lors de sa réunion en 2014.

"Quand j'ai écouté du gwoka pour la première fois, j'ai cru que cette musique venait d'Afrique", explique Gino Sitson, chanteur, compositeur et ethnomusicologue camerounais, élevé en France et vivant à New York. Son intérêt pour cette musique l'entraîne à faire du gwoka son sujet de thèse à la Sorbonne. "En 2010, j’ai commencé par explorer le gwoka de manière scientifique, sous l'angle de l'héritage africain. Ma thèse porte sur le processus de transmission. Je m'appuie sur deux des valeurs esthétiques essentielles à cette musique: l'éloquence et le sentiment. Je fais intervenir l'analyse musicale. Je m'intéresse à la prosodie, à des éléments de sciences cognitives comme la perception de la mémoire, à ce qui a été transmis, au sens de ce jeu musical sur la voix qu'est le 'boula dgel' ['boula' est le nom d'un tambour, 'dgel' est la prononcia- tion de "gueule" en créole : on peut ainsi traduire par 'tambour de bouche']. Il s'agit d'une forme de percussions vocales, un jeu de gorge qui se joue à plusieurs, particulièrement lors des veillées mortuaires [véyé en créole]. Les spécialistes de ce style les plus connus en Guadeloupe sont les membres du groupe Kan'nida, avec lesquels j'ai travaillé. Nous avons enregistré ensemble un album dédié à leurs grands-parents, parents et frères disparus, Ta Yo. J'ai eu la chance de me trouver en immersion totale dans cette culture maintenue vivante par la famille Geoffroy, à l'origine du groupe."

Kan'nida perpétue la tradition d'un gwoka rural des plus authentiques. Cet héritage, qui a perduré tout en se transformant depuis l'abolition de l'esclavage, s'accorde encore au quotidien de cette famille guadeloupéenne. Pour tous les membres de Kan'nida, la musique est une passion, pas un métier... "Il n'y a pas d'artiste professionnel dans notre groupe, explique son leader René Geoffroy. Nous avons un local [dans la ferme familiale] où l'on répète les mardi et jeudi quand nous devons partir en tournée, seulement le jeudi si l'on n'en a pas afin de rester ensemble. Dans notre famille, on a été élevé dans ce milieu. Ce que nous faisons ne nous est pas étranger. C'est en nous, contrairement à beaucoup d'autres Guadeloupéens qui disent que leurs parents les frappaient pour qu'ils n'aillent pas dans les 'lewoz' [soirées festives de gwoka] ou les 'véyé. Nous étions plutôt montrés du doigt : 'Voilà un petit Geoffroy, c'est un 'vié nèg'!", ce qui veut dire chez nous un "vagabond". Je me souviens même qu'un dimanche matin les 
Malou et Zagalo Geoffroy

(c) FRANÇOIS BENSIGNOR

gendarmes sont venus à la maison chercher mon grand frère Sergius et ils l'ont enfermé pendant un jour entier pour qu'il n'aille pas chanter dans une 'véyé'... C'est l'Église qui demandait à la loi de nous intimider... Mais ça n'a pas pris. Parce que cette musique fait partie de la vie des Guadeloupéens. Bien véhiculé, avec tout le respect qu'on lui doit, le gwoka reste une force spirituelle."

\section{Une affaire de famille}

"Notre mère chantait et dansait, poursuit René Geoffroy. Notre père faisait le 'boula dgel.' Tout le monde chantait naturellement. Notre grand frère Sergius [à présent disparu], chantait à 14 ans. Mon frère Francky, dit Zagalo, avait déjà une réputation de footballeur en 1979, lorsqu'il a fondé Kan'nida avec ses souurs Viviane, Brigitte et Christiane (dite Malou). Je suis revenu de métropole en 1981 et, comme j'avais un peu plus de connaissances musicales, ils m'ont demandé de prendre la direction artistique du groupe. Nous avons enregistré un premier 33 tours, La Guadeloupe Malad. En 1982, mon frère Sergius est rentré de métropole et nous avons enregistré un second 33 tours avec lui, Lewoz La, qui a vraiment bien marché. Sergius était très connu et respecté pour sa façon de chanter très personnelle. Il menait aussi ses affaires de son côté. Quand il venait faire des concerts avec nous, on appelait le groupe Kan'nida Plus, parce que c'était vraiment autre chose.

"Pendant une période, nous étions quatre frères et trois sceurs sur les onze membres du groupe. Nous avons malheureusement perdu deux de nos frères, 


\section{MUSIQUES}

qui ont été remplacés. Au début, je ne chantais pas, mais leur disparition m'a incité à le faire, parce qu'on a besoin de deux chanteurs leaders, deux couleurs différentes pour bien véhiculer notre tradition. Les personnes qui nous accompagnent sont des voisins, qui se reconnaissent dans ce qu'on fait. Notre relation est très solidaire. C'est comme une grande famille recomposée!

"Nous habitons en pleine campagne. Quand on va attacher les boufs, on peut crier tant qu'on veut. Les paroles de nos chansons racontent notre vécu. C'est ce que nous aimerions montrer au reste du monde, parce que le simple fait d'écouter l'histoire de Kan'nida pourrait servir de thérapie, comme un repère de simplicité pour aborder la vie de tous les jours. Ce que nous faisons est une musique à part entière. On est né dedans et on sait pourquoi on la fait. On sait aussi qu'on est en train de véhiculer quelque chose qui était là. On fait un bout de chemin avec et après, d'autres poursuivront. On est persuadé qu'on est dans la bonne voie, parce qu'on essaye de le faire le plus simplement possible, avec beaucoup de force de conviction. On garde toujours la joie de le faire. On n'a pas une vie avec la musique et une vie ailleurs : le gwoka fait partie de notre vie. C'est pour ça qu'on est bien dedans."

\section{Le patrimoine culturel immatériel}

L'inscription du gwoka à l'Unesco, on la doit avant tout à un homme de passion, Félix CotelIon. Avocat de métier, bâtonnier de 2005 à 2007, il a su tout mettre en œuvre pour redonner au gwoka sa valeur intrinsèque. On lui doit la fondation du Festival de gwoka de Sainte-Anne, dont la première édition a eu lieu en juillet 1988. II est aussi le principal responsable de la création de l'ONG Repriz, Centre régional des musiques et danses traditionnelles et populaires de Guadeloupe, qu'il préside depuis sa création en juil- let 2005. L'inscription du gwoka sur la liste représentative du patrimoine culturel immatériel de l'humanité de l'Unesco est un projet qu'il mûrit patiemment depuis de nombreuses années. Félix Cotellon nous en retrace les différentes étapes: “Depuis les années 2000, j'ai suivi régulièrement les colloques qu'organisait Cherif Khaznadar à la Maison des cultures du monde. Ils ont joué un rôle très important dans la préparation de la démarche d'inscription du gwoka. Le premier rendez-vous de la Guadeloupe autour de la convention élaborée parl'Unesco en 2003 s'est déroulé l'année suivante dans le cadre du Festival de gwoka de Sainte-Anne. Auparavant, nous avions initié le projet Lyannaj [qui traduit les notions de lien, d'union, de collectif, d'être ensemble], qui consistait à travailler sur les apports culturels de l'Europe, de l'Afrique et de I'Inde en Guadeloupe. Ces thèmes étaient ceux des rencontres organisées pendant le festival en 2001, 2002 et 2003. Et, pour finaliser ce cycle sur la diversité culturelle, nous avons accueilli Cherif Khaznadar en 2004, afin qu'il nous parle de la convention et de la déclaration universelle de l'Unesco sur la diversité culturelle.

"Cette première démarche n'avait pas été entreprise en vue de l'inscription du gwoka sur la liste représentative du $P C l$. Mais je me suis rendu compte que la convention pouvait effectivement permettre aux institutions de prendre connaissance des recommandations qui avaient déjà été prises au niveau international. Elles pouvaient aussi constater que nous n'avions pas attendu cette convention pour commencer à sauvegarder notre patrimoine - au même titre que la plupart des porteurs de traditions."

\section{La liste représentative du PCI}

Félix Cotellon : "À partir de 2008, j'ai impulsé la démarche d'inscription du gwoka par un travail d'information et de sensibilisation : séminaires, 
réunions, etc. Nous avons présenté la convention et ouvert des débats en posant cette question : 'Fautil inscrire le gwoka sur la liste représentative?' Repriz a organisé plusieurs réunions d'information, afin de sensibiliser le public sur la convention. II n'était pas question pour nous de ne pas nous référer au préalable à la majorité des Guadeloupéens, pour qui le gwoka occupe une place très importante en terme de patrimoine vivant. Nous avons fait de ce débat une question sociétale en interrogeant l'ensemble de la société guadeloupéenne.

"Dans un premier temps, l'information sur la convention ne suscitait pas vraiment de questions. C'est lorsque nous avons décidé de mener à bien l'inscription que des voix se sont élevées. II fallait mettre en place une méthode. En 2011, Repriz a lancé un appel à tous les porteurs de tradition, expliqué la démarche qui consistait à suivre les recommandations de l'Unesco, puis constitué un groupe de travail scindé en plusieurs cellules. La cellule identification était chargée de décrire ce qu'est le gwoka. La cellule information organisait des réunions sur tout le territoire guadeloupéen. La cellule sauvegarde travaillait sur les mesures que nous estimions nécessaires pour le gwoka. Avec la cellule financière, ces différentes commissions se sont mises au travail. Nous avons intitulé ce groupe Comité lyannaj pou gwoka'. Repriz est devenu membre de ce comité au même titre que les autres associations qui y participaient. Une quarantaine de personnes, porteurs de traditions et associations, le constituaient.

"C'est au cours du travail d'information que sont apparus les premiers différends. Une quarantaine de réunions ont eu lieu sur l'ensemble du territoire, accompagnées d'une importante communication via les médias. L'ordre du jour comprenait deux parties : présentation de la convention, intérêt de l'inscription du gwoka pour la Guadeloupe.
Le Festival de gwoka 2011 a pris pour thème 'linscription du gwoka sur la liste représentative de I'Unesco'. Chaque soir sur scène, devant le public, les points clés étaient abordés durant une quinzaine de minutes. Et une brochure expliquant le projet a été largement diffusée."
Félix Cotellon et René Geoffroy (c) François Bensignor

\section{Les contestations}

Félix Cotellon: "La question a suscité un grand débat. Pendant un an, toutes les couches sociales (syndicats, organisations politiques, etc.) se le sont approprié. Des réserves, des craintes sont apparues. Certains porteurs de traditions ont dit que pour eux, cette inscription n'avait aucun intérêt. Certains disaient: 'Nous transférons notre patrimoine à l'Unesco, qui aura la mainmise dessus et en décidera.' D'autres disaient : 'Le gwoka est vivant et on ne voit pas pourquoi il faudrait le sauvegarder.' 


\section{MUSIQUES}

Des questions ont également été soulevées autour de la 'propriété intellectuelle', qui était transférée à l'Unesco et, par ce biais, à la France. Ce sont les questions clés qui ont été portées au débat, parallèlement à celle mettant en avant que le gwoka a surtout besoin d'être mieux diffusé.

"Nous avons expliqué la notion de sauvegarde, qui prête souvent à confusion, en précisant la définition qu'en donne la convention de l'Unesco. II s'agit d'assurer la viabilité du patrimoine, ainsi que sa documentation, la recherche dont il peut faire l'objet, sa diffusion, sa promotion, etc. Nous avons montré qu'en aucun cas l'Unesco ne pouvait s'approprier un patrimoine qui appartient aux porteurs de traditions, qui le transmettent et sans lesquels ce patrimoine n'est plus vivant. Nous avons fait valoir qu'il serait bien étrange que l'Unesco aille à l'encontre de ses propres recommandations, selon lesquelles ce sont les communautés humaines qui font vivre les patrimoines, qui périraient sans elles. Quant à la question de la propriété intellectuelle, il y avait aussi un malentendu, que nous avons dissipé. L'inscription sur une liste de l'Unesco ne doit pas être assimilée à celle d'une ceuvre à l'Institut national de la propriété industrielle (INPI). Nous avons expliqué qu'il ne s'agit nullement de transmettre à I'Unesco la propriété des ceuvres, sur lesquelles ils possèdent un droit inaliénable.

"D'autres questions ont également été soulevées. Par exemple: 'Comment se fait-il que toi, Cotellon, anticolonialiste avéré, tu laisses la France présenter le projet à l'Unesco ?'C'est une question éminemment politique et j'y ai répondu. 1 : La Guadeloupe est un département français. 2 : Les Guadeloupéens ont manifesté leur volonté de rester dans le cadre de la République française à l'occasion de deux référendums. 3 : La grande majorité des organisations anticolonialistes ont un projet politique institutionnel pour la Guadeloupe, qui est la créa-

Félix Cotellon

(c) François Bensignor

tion d'une nouvelle collectivité dans le cadre de la République française. Et dans l'éventuelle mise en place de cette nouvelle collectivité, la politique extérieure fait partie des compétences laissées à la France. Je ne vois donc aucun problème à ce que ce soit la France qui présente l'inscription du gwoka, puisque celle-ci entre dans le cadre de la politique internationale."

\section{Une consultation démocratique}

Félix Cotellon : "Pour le dépôt de la demande d'inscription, l'Unesco recommande l'obtention du consentement préalable, libre et éclairé des communautés, des groupes et des individus. Nous avons donc recueilli plus de 4000 signatures sur une pétition en faisant la demande. L'information a circulé à travers tous les médias. Nous avons sensibilisé toutes les collectivités territoriales et avons obtenu l'assentiment du Conseil régional, de tous 
les sénateurs, de tous les députés. Dans les 30 engagements du candidat François Hollande à l'élection présidentielle figurait l'inscription du gwoka sur la liste représentative du patrimoine culturel immatériel de l'humanité de l'Unesco. Nous avons initié des réunions à Paris et à Londres avec la communauté guadeloupéenne et mis en place un site Internet.

"Durant les réunions des cellules, nous avons recueilli toutes les propositions de mesures de sauvegarde que les porteurs de traditions jugeaient important de faire figurer au dossier de candidature. Celles-ci ont été publiées dans un document intitulé Nou ka Travay! : Je crois que c'est la seule initiative de cette nature qui ait été faite sur le territoire français. En Corse et en Bretagne [dont respectivement le Cantu in Paghjella et le fest-noz ont été inscrits sur la liste représentative du PCI], les méthodes pour constituer les dossiers de candidature n'ont pas été aussi démocratiques.

"Dans de nombreux cas, ce sont les institutions - régions, provinces, États, etc. - qui pilotent les dossiers de l'Unesco. Le cas de la Guadeloupe est différent: ce sont les Guadeloupéens, représentant toutes les catégories sociales, qui sont à l'initiative de la demande d'inscription. Les collectivités territoriales ont joué un rôle très secondaire. Un premier dossier a été déposé à l'Unesco en 2012. Or la liste représentative a rencontré un tel succès que l'Unesco a dû limiter les demandes à une candidature par pays et par an. Le dossier du fest-noz breton ayant été déposé au préalable, la France a dû attendre une nouvelle session. Le dossier du gwoka, réadapté en fonction de l'évolution des critères de l'Unesco, a donc été déposé en avril 2013 au ministère de la Culture, qui l'a sélectionné parmi d'autres dossiers. II a fait partie des 60 dossiers - dont 47 demandes d'inscription sur la liste représentative - examinés par l'Unesco lors de sa neuvième session de novembre 2014.

"L'inscription du gwoka revêt d'abord un intérêt pour la Guadeloupe. Elle incitera les pouvoirs publics à prendre conscience de l'importance du gwoka et à en tenir compte dans leurs politiques de développement culturel, notamment en matière de patrimoine culturel immatériel. Elle renforcera l'image et l'attractivité de la Guadeloupe. Elle pourra également favoriser de nouvelles coopérations culturelles avec les pays de la Caraïbe, voire de l'Amérique centrale et de l'Amérique du Sud."

Le 28 octobre à Paris, le Comité intergouvernemental de sauvegarde du patrimoine culturel immatériel de l'Unesco publiait le rapport de l'Organe subsidiaire sur ses travaux en 2014 et examen des candidatures pour inscription sur la Liste représentative du PCI de l'humanité. À la page 39 du rapport, le projet de décision concernant le gwoka se conclut en ces termes : "Le Comité (...) inscrit le gwoka: musique, chants, danses et pratique culturelle représentatifs de l'identité guadeloupéenne sur la Liste représentative du patrimoine culturel immatériel de l'humanité." -

\section{Repriz : http://repriz-cmdtp.org/}

\section{Discographie :}

Kan'nida :

Ta Yo (2013) - Nou Ka Travay (2010)

Léwoz (2008) - Bèlè-bodé (2006)

Kyenzann (Indigo/Label Bleu, 2000)

Vis an nou (Indigo/Label Bleu, 1996)

Ye, jodi, demen (1995) - Mizik en nou (1993)

Kan'Nida (1992)

Gino Sitson :

Voi Strings (Buda Musique, 2014)

Listen (Alessa Records - Buda Musique /

Universal, 2013) 